\title{
Testing aspheric surfaces with computer-generated holograms: analysis of adjustment and shape errors
}

\author{
Bernd Dörband and Hans J. Tiziani
}

\begin{abstract}
Various interferometric methods can be used for testing aspheric surfaces with high accuracy. Using a partially compensating lens in a Twyman-Green arrangement together with a computer-generated hologram instead of a null corrector gives great flexibility. For quantitative analysis a high-resolution automatic fringe analysis is necessary. The interferometric arrangement and the setup for the interferogram data acquisition are described along with different algorithms for calculating the wave front data. Tilt and decentering of the aspheric surface and the synthetic hologram as well as the actual aspherical parameters can be derived from the calculated wave front using Zernike polynomials to communicate with a ray tracing program. For small adjustment and shape errors the linearity will hold, leading to fast correction and numerical compensation of their effects on the surface error. The method is verified by several examples. Experimental results agree with the proposed model.
\end{abstract}

\section{Introduction}

Aspheric surfaces will be used more frequently when manufacturing and testing in industrial environments can be improved. In addition to a point by point analysis with optical, mechanical, or inductive testers, interferometric techniques are frequently used leading to faster and more accurate results of the test surface's shape error. Many different interferometric methods are used such as testing the complete optical system or using a null corrector in the test arm of a TwymanGreen interferometer to compensate the asphericity of the surface under test. Shearing interferometry or holography, where a hologram of a master surface serves as a reference, also is among the techniques used.

A promising method is the use of computer-generated holograms (CGHs) together with a partially compensating lens to compare the wave front of an aspheric test surface to a perfect one generated by a synthetic hologram in a two-beam interferometer. Alternatively, the aspheric wave front can be used to reconstruct a plane wave front modulated with the surface errors which in turn are compared with a perfect reference wave.

The authors are with Universität Stuttgart, Institut für Technische Optik, Pfaffenwaldring 9, D-7000 Stuttgart 80, Federal Republic of Germany.

Received 29 December 1984.

0003-6935/85/162604-08\$02.00/0.

(C) 1985 Optical Society of America.
A CGH can be used for testing a wide range of aspheric surfaces with high accuracy leading to an absolute measure of the surface errors. By contrast, in a relative test a master surface needs to be available.

A number of papers on testing aspheric surfaces with CGHs have been written.1-14 From a practical point of view a two-beam arrangement in which both beams pass through the CGH is desirable. In this way the inhomogenities of the hologram storage material, which often is a photographic plate, are compensated. For symmetrical optical systems to be tested, in-line (Gabor-type) and off-axis CGHs can be used. ${ }^{14} \mathrm{~A}$ two-wavelength technique reduces the sensitivity. ${ }^{15}$

Implementation of the interferometric technique requires the analysis of possible errors due to imperfect optical interferometer components. An automatic fringe analysis is useful to calibrate the interferometer with a well-known perfect spherical surface and to measure surface deviations with resolutions of $\lambda / 100$. Adjustment of the interferometric setup can be timeconsuming; automatic fringe analysis is desirable if high precision is required. In the following the testing procedure with CGHs, the fringe analysis algorithms, and a method for computer detection of adjustment and shape errors along with their numerical compensation are described.

\section{Testing Procedure with Computer-Generated Holograms}

A two-beam interference arrangement of the Twyman-Green type with an off-axis hologram for testing 
aspheric surfaces is shown in Fig. 1. The incident wave is separated by the beam splitter into test and reference beams, reflected back from the mirror $M$ slightly obliquely, to be used as the reference by passing through the hologram undisturbed. For fringe analysis of closed fringes a plane-parallel plate is used to shift the phase in steps of $90^{\circ}$. The simple lenses $L_{1}, L_{2}$, and $L_{3}$ are auxiliary lenses to adapt the aperture of the lens under test TS. They should also image the test surface onto the $\mathrm{CGH}$, a necessary condition for strong aspheric wave fronts and should compensate some of the wave front's asphericity. $L_{4}$ is a high quality lens to be used for testing spherical surfaces without a CGH by replacing the auxiliary lenses $L_{1,2,3}$ by $L_{1}^{\prime}$. In addition $L_{4}$ and $L_{1}^{\prime}$ are used for focusing on the aspheric's vertex to have a precise reference point when placing the surface into the correct testing position. A spatial filter in the focus plane of $L_{5}$ avoids unwanted light; $L_{6}$ images the fringe pattern as a measure of the discrepancy between the test piece and the perfect aspheric onto a diode array or a video camera connected to a computer for fringe analysis. For testing aspheric surfaces the separation of lenses $L_{1}, L_{2}$, and $L_{3}$ may need to be modified or one or more lenses may be replaced appropriately. They are by no means perfect; they need to be tested by replacing the aspheric surface by a known spherical mirror. For perfect agreement no fringes should occur. Sources of errors result from the departure of lens shapes and separations used for computing the wave front as well as for centering errors of the components, magnification error and distortion by photoreduction, and errors in generating the hologram. Most of the errors can be compensated by an appropriate hologram containing additional correcting terms in the actual wave front.

For generating the computer hologram we use an Optronics drum plotter with a minimum step length of $12.5 \mu \mathrm{m}$, thus allowing 20,000 by 20,000 pixels to be plotted onto $25 \times 25 \mathrm{~cm}^{2}$ of photographic film in 256 different gray levels. For final use in the interferometer a one-step reduction on high contrast photographic plates or on photoresist is required. The latter is used when improved diffraction efficiency is necessary.

\section{Automatic Fringe Analysis}

The fringe patterns obtained by interferometry represent contour lines of phase differences between the interfering wave fronts. In the Twyman-Green interferometer they contain information about the shape error of the surface under test. A quantitative evaluation of the fringe pattern thus leads to a quantitative description of the surface error.

For fringe analysis many different methods are used. They can be classified into static, where a single interferogram (photograph, video image) is analyzed, or dynamic, where the fringe pattern is manipulated to get a phase change in time.

\section{Static algorithms are}

Analysis "by hand" using ruler and pencil to determine the position of fringer centers or analysis using a digitizing tablet. ${ }^{16,17}$

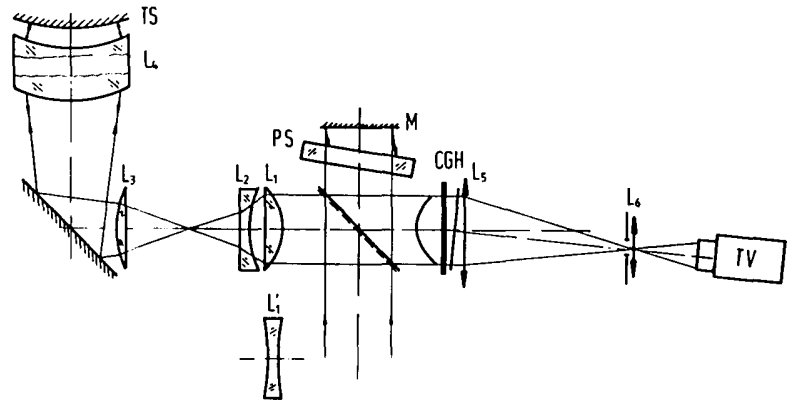

Fig. 1. Twyman-Green interferometer with a CGH to test aspheric surfaces.

Localization of fringe centers using video techniques and image processing. ${ }^{18-22}$

Phase detection technique in the spatial domain using Fourier transformation ${ }^{23}$ or Fourier analysis in connection with a video technique..$^{24,25}$

Nearly all static methods need fringe patterns with monotonous increases of fringe orders which are achieved by tilting the interferometer's reference mirror. Using the Fourier techniques, strong tilts are desirable to arrive at high carrier frequencies.

Dynamic algorithms are

Phase stepping by changing the phase difference between the two interfering beams by a certain amount and measuring the intensities at selected sample points for each step. ${ }^{26}$ Three steps are a minimum, changing the phase by $90^{\circ}$ or $120^{\circ}$ per step. ${ }^{27,28}$ Using four steps leads to a simple mathematical expression. ${ }^{29}$

Heterodyne techniques using two different wavelengths which lead to periodic changing intensities in the interference pattern with phase delays according to the optical path differences. Since phase shifting is not interrupted during sampling the use of integrating detectors is recommended using the "integrating bucket" calculation similar to the phase stepping methods. ${ }^{30}$

The ac-phase detection technique involve a timevarying manipulation of the reference phase combined with electronic phase detection of the varying intensity signal similar to the heterodyne technique. However, the change of the reference phase is not necessarily linear in time. Applying a sinusoidal movement to the reference mirror includes the possibility for phaselocked interferometric systems. ${ }^{31}$

All dynamic methods can calculate the phase difference at any point within the interference pattern and allow analysis of the closed fringes. Inhomogeneous illumination of the interferometer as well as different sensitivities of the detector elements have no influence on the measurement.

\section{Fringe Analysis Setup and Applied Algorithms}

For the phase measurement two different systems have been established:

A Hamamatsu C 1000 camera connected to a DEC PDP 11/34 computer including the necessary software to provide control over camera functions and the phase stepping device. The latter consists of a Burleigh Inch-Worm Translator which slightly rotates a plane- 

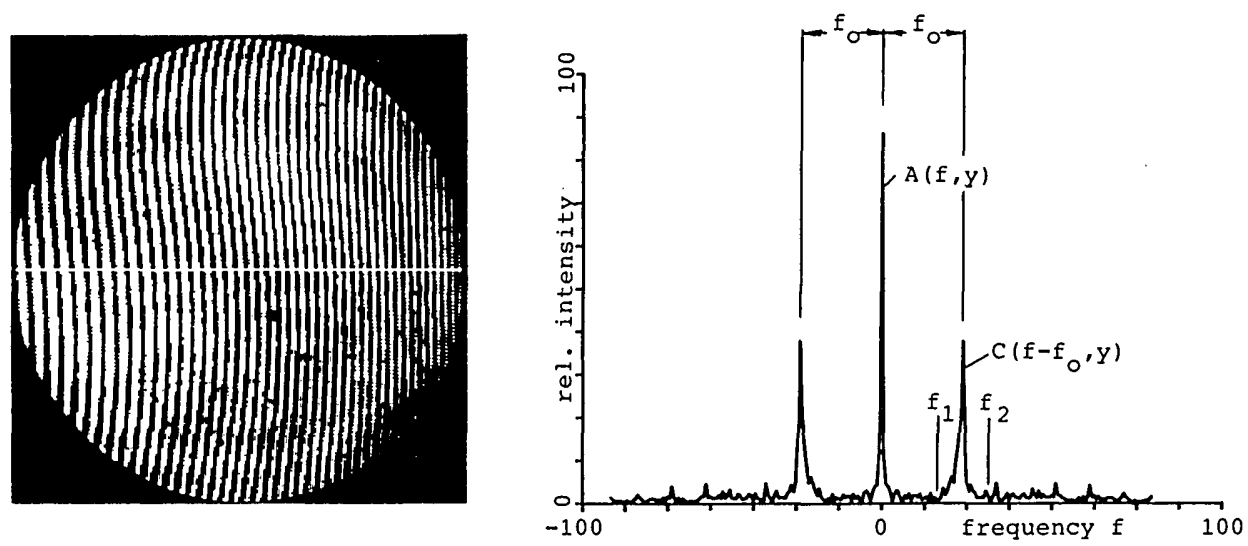

Fig. 2. Fourier spectrum of fringe pattern with high carrier frequency.

parallel plate within the reference arm of the interferometer. The camera provides up to $1024 \times 1024$ data points containing 256 gray levels. Special columns and lower resolutions can be selected.

A Fairchild 1500 R-CCD line scan camera with 2048 pixels connected to an IBM-PC computer by an adequate controller. The computer is equipped with graphic and $\mathrm{D} / \mathrm{A}-\mathrm{A} / \mathrm{D}$ facilities. Phase stepping could also be obtained by analog output to a ramp generator, which in turn controls a piezoelectric-driven reference mirror.

For automatic fringe analysis we use the spatial phase detection technique based on a FFT algorithm ${ }^{23}$ as well as the three-phase step method. ${ }^{27,28}$ Both of them are now briefly described.

\section{A. FFT Method}

A strong tilt between test and reference waves has to be introduced to produce an intensity pattern $I(x, y)$ :

$$
I(x, y)=a(x, y)+b(x, y) \cos [\phi(x, y)+k x \sin \theta],
$$

with $\quad a(x, y)=$ average intensity, $b(x, y)=$ modulation, $\phi(x, y)=$ phase difference to be detected, and

$k=(2 \pi) / \lambda=$ wave number.

Introducing complex notation leads to

$$
\begin{aligned}
I(x, y)= & a(x, y)+c(x, y) \exp (i k x \sin \theta) \\
& +c^{*}(x, y) \exp (-i k x \sin \theta),
\end{aligned}
$$

with $c(x, y)=1 / 2 b(x, y) \exp [i \phi(x, y)]$ and $c^{*}(x, y)=$ $1 / 2 b(x, y) \exp [-i \phi(x, y)]$ as the conjugate complex. Fourier transformation in the $x$ direction leads to

$$
\tilde{I}(f, y)=A(f, y)+C\left(f-f_{0}, y\right)+C^{*}\left(f+f_{0}, y\right) \text {, }
$$

with $\tilde{I}, A$, and $C$ as Fourier transforms of the variables $I, a$, and $c$.

Choosing an adequate carrier frequency will give three peaks in the spectrum which can be easily separated (Fig. 2). When $C\left(f-f_{0}, y\right)$ is filtered, shifted to the origin, and transformed back, one arrives at

$$
F^{-1}\{C(f, y)\}=c(x, y)=1 / 2 b(x, y) \exp [i \phi(x, y)] .
$$

$\phi(x, y)$ can be obtained by either

$$
\phi(x, y)=\arctan \frac{\operatorname{Im}\{c(x, y)\}}{\operatorname{Re}\{c(x, y)\}}
$$

or applying the complex natural logarithm:

$$
\ln c(x, y)=\ln \frac{b(x, y)}{2}+i \phi(x, y)
$$

containing $\phi(x, y)$ entirely within the imaginary part.

\section{B. Three-Phase Step Method}

The three intensity patterns that differ by phaseshifts of $\Delta$ can be expressed as

$$
\begin{aligned}
& I_{1}(x, y)=a(x, y)+b(x, y) \cos [\phi(x, y)-\Delta], \\
& I_{2}(x, y)=a(x, y)+b(x, y) \cos \phi(x, y), \\
& I_{3}(x, y)=a(x, y)+b(x, y) \cos [\phi(x, y)+\Delta] .
\end{aligned}
$$

Using addition theorems we can arrive at

$$
\phi(x, y)=\arctan \left[\frac{I_{3}-I_{1}}{I_{1}+I_{3}-2 I_{2}} \cdot \tan \frac{\Delta}{2}\right] .
$$

Using $\Delta=90^{\circ}$ results in the simplest case of $\tan \Delta / 2=$ 1 ; however $\Delta=120^{\circ}$ is sometimes practiced arriving at $\tan \Delta / 2=\sqrt{3}$ requiring more calculation.

\section{Compensation of Adjustment Errors}

Adjustment of the interferometric setup can be time-consuming, since seven degrees of freedom need to be balanced: tilt and decentering of the aspheric surface in two directions and decentering as well as rotation of the hologram. A lot of skill and patience is needed, especially since some of the adjustment errors have similar effects on the interference pattern.

We have found it appropriate to approximate the measured wave front by a set of polynomials; Zernike polynomials were used because of their orthogonal properties. They easily interface with the ray tracing program to calculate and eliminate the influences of the actual adjustment errors on the measured wave front. 


\begin{tabular}{lll}
\hline \multicolumn{1}{c}{$y$ Direction } & $x$ Direction \\
\hline$U_{2}=r \sin \theta$ & $U_{3}=r \cos \theta$ \\
$U_{8}=\left(3 r^{3}-2 r\right) \sin \theta$ & $U_{9}=\left(3 r^{3}-2 r\right) \cos \theta$ \\
$U_{19}=\left(10 r^{5}-12 r^{3}+3 r\right) \sin \theta$ & $U_{19}=\left(10 r^{5}-12 r^{3}+3 r\right) \cos \theta$ \\
$U_{32}=\left(35 r^{7}-60 r^{5}+30 r^{3}-4 r\right) \sin \theta$ & $U_{33}=\left(35 r^{7}-60 r^{5}+30 r^{3}-4 r\right) \cos \theta$ \\
\hline
\end{tabular}

Let $w(r, \theta)$ be an approximation of the measured wave front $W(r, \theta)$ :

$$
W(r, \theta) \cong w(r, \theta)=\sum_{j=0}^{N} A_{j} U_{j}(r, \theta),
$$

with $A_{j}$ as coefficients and

$$
U_{j}(r, \theta)=U_{n}^{\dot{m}}(r, \theta)=R_{n}^{m}(r)\left\{\begin{array}{l}
\sin \\
\cos
\end{array}\right\} m \theta
$$

as Zernike polynomials; $r$ and $\theta$ are radius and angle within the unit circle; and $m$ and $n$ are whole numbers with $n$ representing the polynomial degree and $m$ representing the azimuth frequency. Furthermore

$$
n \geq 0,-n \leq m \leq n \quad(n-m \text { is an even number). }
$$

For $m \geq 0$ the cosine function, for $m<0$ the sine function is valid.

$$
j=\frac{n(n+2)-m}{2}+1
$$

is the number of the polynomial term starting with $j=$ 1 for $n=m=0$, and $R_{n}^{m}(r)$ is defined as

$$
R_{n}^{m}(r)=\sum_{s=0}^{\frac{n-m}{2}}(-1) s \frac{(n-s) !}{s !\left(\frac{n+m}{2}-s\right) !\left(\frac{n-m}{2}-s\right) !} r^{(n-2 s)} .
$$

Taking into account that mainly odd terms will arise when decentering and tilting the optical elements by only a small amount, polynomials with $m= \pm 1$ need to be evaluated, whereas defocusing and errors in radius and eccentricity are described by rotational symmetric polynomials with $m=0$. Since rotation of an off-axis hologram leads only to a linear part in the wave front, which is compensated by a tilt of the reference mirror, there are at least four odd terms to be evaluated for the $x$ and $y$ directions, respectively, as shown in Table $\mathrm{I}$.

Calculating the derivatives of the Zernike coefficients $A_{2} \ldots A_{33}$ with respect to decentering and tilt of the aspheric surface and the hologram by ray tracing leads to a set of linear equations:

$\left(\begin{array}{l}A_{2} \\ A_{3} \\ A_{8} \\ A_{9} \\ A_{18} \\ A_{19} \\ A_{32} \\ A_{33}\end{array}\right)=\left(\begin{array}{cccc}\frac{\partial A_{2}}{\partial \beta_{R x}} & \frac{\partial A_{2}}{\partial \beta_{R y}} \ldots \frac{A_{2}}{\partial \epsilon_{H x}} & \frac{\partial A_{2}}{\partial \epsilon_{H y}} \\ \vdots & & \cdot \\ \vdots & & \cdot \\ & \cdot & & \cdot \\ \frac{\partial A_{33}}{\partial \beta_{R x}} & \frac{\partial A_{33}}{\partial \beta_{R y}} \ldots \frac{\partial A_{33}}{\partial \epsilon_{H x}} & \frac{\partial A_{33}}{\partial \epsilon_{H y}}\end{array}\right) \cdot\left(\begin{array}{c}\beta_{R x} \\ \beta_{R y} \\ \epsilon_{A x} \\ \epsilon_{A y} \\ \beta_{A x} \\ \beta_{A y} \\ \epsilon_{H x} \\ \epsilon_{H y}\end{array}\right)$,

(13) which has to be inverted to calculate the amounts of tilt and decentering.

. Here $\beta_{R x / y}$ is the tilt of reference mirror or rotation of off-axis hologram,

$\beta_{A x / y}$ is the tilt of the aspheric surface,

$\epsilon_{A x / y}$ is the decentering of the aspheric surface,

$\epsilon_{H x / y}$ is the decentering of the hologram,

$A_{2} \ldots A_{33}$ are the measured Zernike coefficients, and

$\left(\partial A_{2}\right) /\left(\partial \beta_{R x}\right)$ are the calculated derivatives $\ldots\left(\partial A_{33}\right) /\left(\partial \epsilon_{H y}\right)$ from ray tracing.

For small adjustment errors linearity can be assumed, so that the inversion delivers accurate results. Stronger errors will need an iterative process such as the damped least-squares method, to arrive at the measured coefficients by ray tracing.

To increase the accuracy more higher-degree coefficients can be added as far as they are significant. In this case the set of linear equations contains more equations than unknown variables, and a least-squares fit algorithm has to be used for the inversion. Once the adjustment errors have been calculated, their contribution to the wave front can be easily determined in terms of Zernike polynomials which can be subtracted from the measured wave front.

The algorithm described above works well assuming an aspheric surface with mainly rotational symmetric errors. Care should be taken when testing surfaces with stronger asymmetric errors. In this case shape errors could be interpreted as adjustment errors leading to an overcompensation of the odd terms.

In Figs. 3-6 some examples of adjustment error compensations are presented. A vertical cross section was evaluated by the automatic fringe analysis program using 1024 pixel resolution. Using the above-described calculation the adjustment errors were determined with respect to the $y$ direction. The $x$ direction was neglected for simplicity. Substracting the odd terms due to decentering and tilt leads to identical curves of shape error.

\section{Calculation of Actual Aspherical Parameters}

Almost the same procedure can be followed to extract information about the shape errors of the aspheric surface under test, such as errors of vertex radius and asphericities. The main difference is in the choice of Zernike terms, since rotational symmetric problems should be solved. Zernike polynomials with $m=0$ up to the 10th degree are listed in Table II. 
a

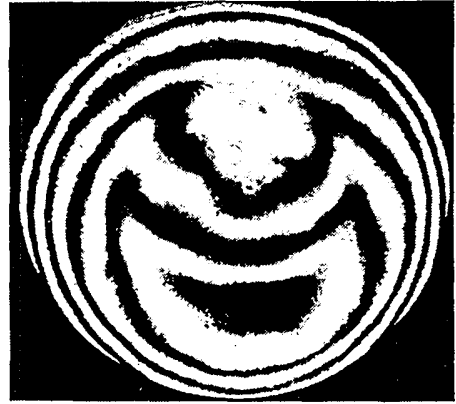

b

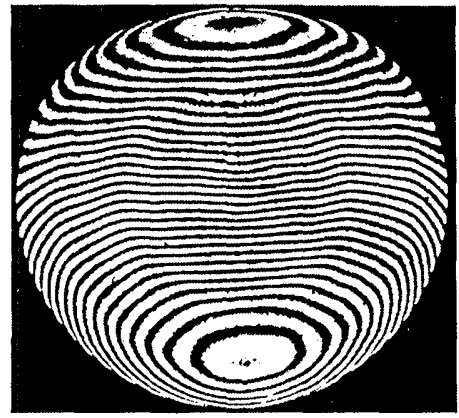

C

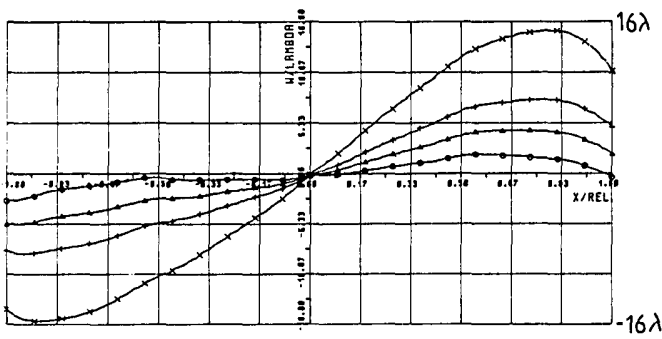

d

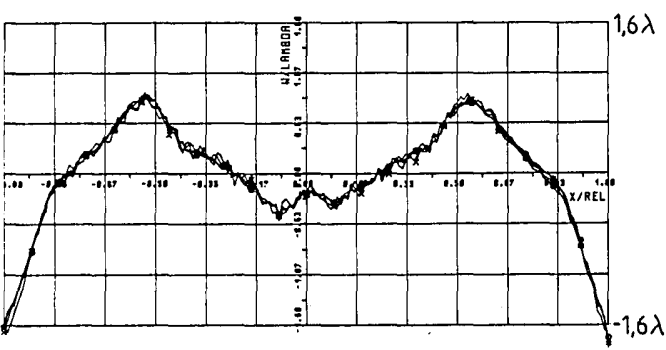

Fig. 3. (a) Interferogram of 0.01 -mm decentered aspheric; (b) interferogram of 0.10 -mm decentered aspheric; (c) vertical cross section through wave fronts for 0.01-, 0.03-, 0.05-, and 0.10-mm decentered aspherics; (d) compensated curves from (c).

a

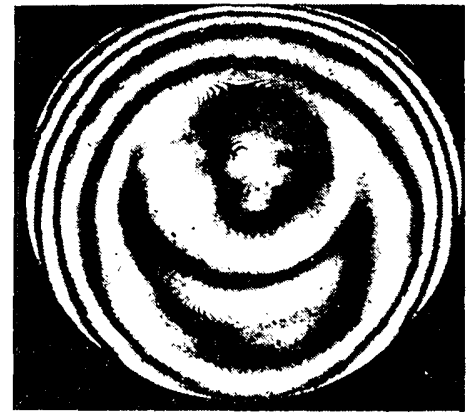

b

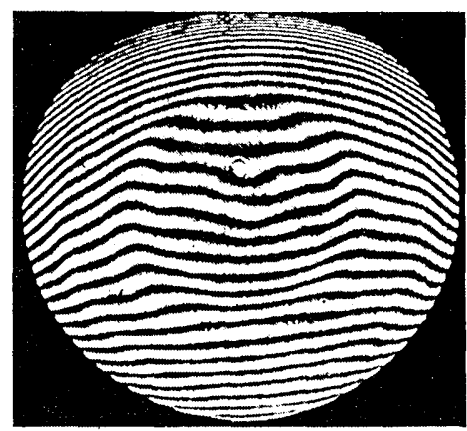

C

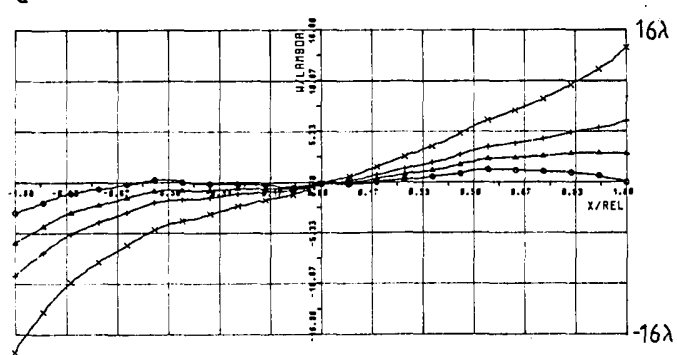

d

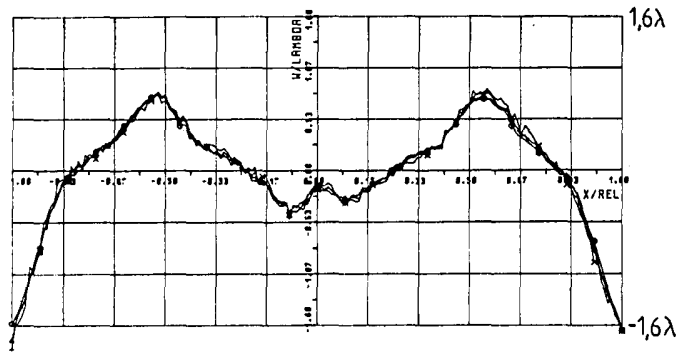

Fig. 4. (a) Interferogram of three units tilted aspheric; (b) interferogram of thirty units tilted aspheric; (c) vertical cross section through wave fronts for three, nine, fifteen, and thirty units tilted aspheric; (d) compensated curves from (c).

For example, calculating the derivatives of the Zernike coefficients with respect to vertex radius $r$ and eccentricity $e$ by ray trace leads to a set of linear equations:

$$
\left(\begin{array}{l}
A_{5} \\
A_{13}
\end{array}\right)=\left(\begin{array}{ll}
\frac{\partial A_{5}}{\partial r} & \frac{\partial A_{5}}{\partial e} \\
\frac{\partial A_{13}}{\partial r} & \frac{\partial A_{13}}{\partial e}
\end{array}\right) \cdot\left(\begin{array}{l}
\Delta r \\
\Delta e
\end{array}\right),
$$

with $A_{5}, A_{13}$ representing the measured coefficients, and $\Delta r$ and $\Delta e$ being the equivalent changes in aspherical parameters according to the linear model. For larger coefficients linearity might not hold, so that optimization techniques must be used. More equations than unknown parameters may be added to increase the accuracy; however, polynomial expansions beyond the 10 th or 12 th degree are hardly ever significant. 
a

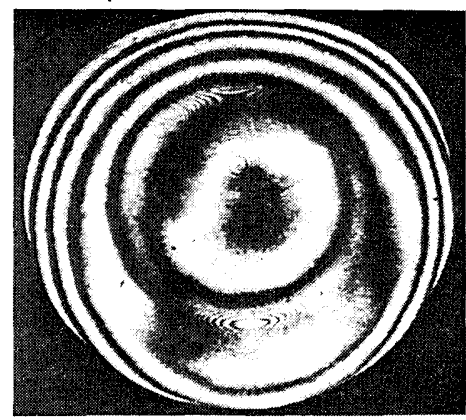

b

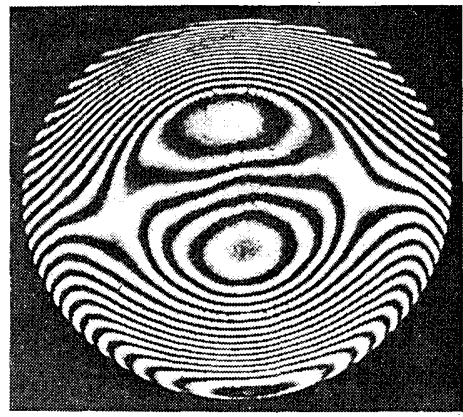

c

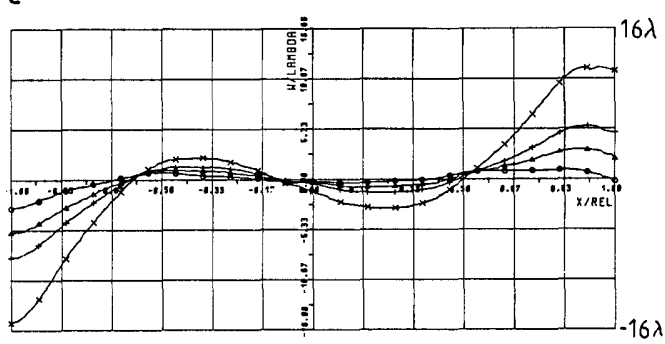

d

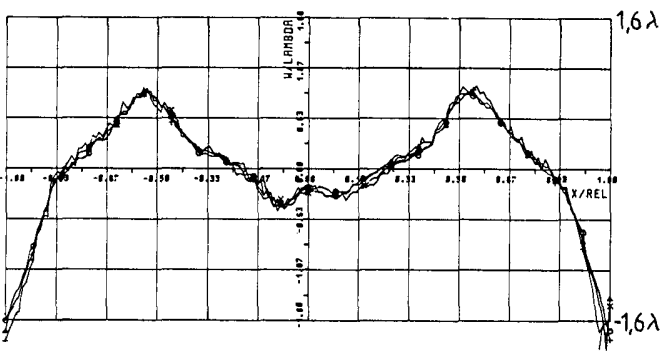

Fig. 5. (a) Interferogram of 0.1-mm decentered hologram; (b) interferogram of 1.0-mm decentered hologram; (c) vertical cross section through wave fronts for 0.1-, 0.3-, 0.5-, and 1.0-mm decentered holograms; (d) compensated curves from (c).

a

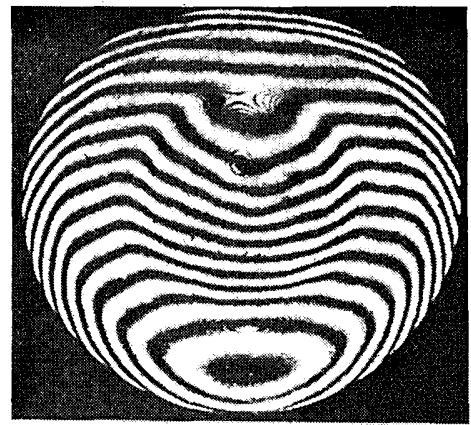

c

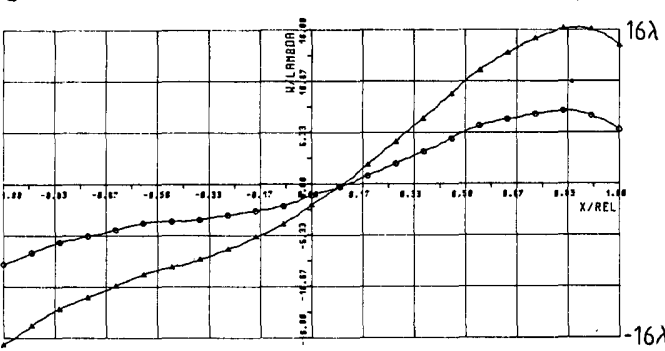

$\mathrm{b}$

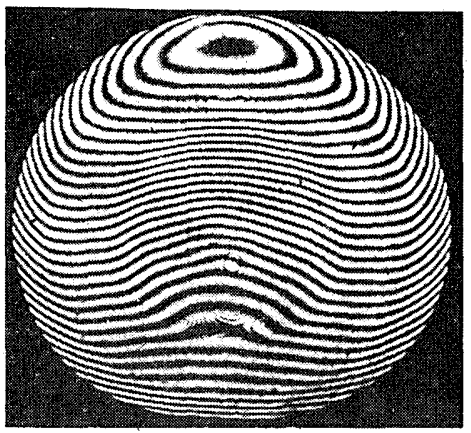

d

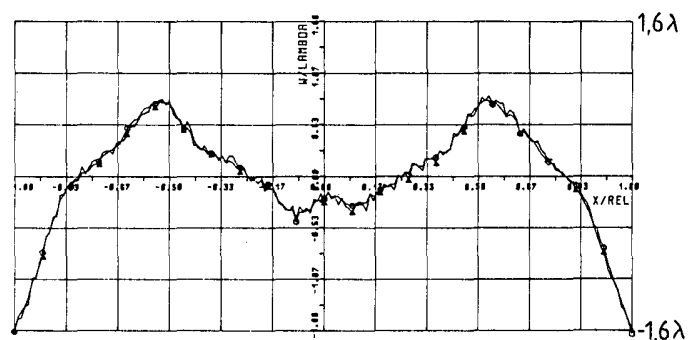

Fig. 6. (a) Interferogram of 0.03-mm decentered and six units tilted aspheric with 0.1-mm decentered hologram; (b) interferogram of 0.06-mm decentere and fifteen units tilted aspheric with centered hologram; (c) vertical cross section through wave fronts from (a) and (b); (d) compensated curves from (c).

Calculating the actual aspherical parameters assumes a perfectly focused aspheric surface, otherwise the effect of defocus will be misinterpreted as shape error.

Figure 7 shows an example of a 1-D and 2-D analysis of an aspheric germanium surface. According to the cross-sectional measurement in Fig. 7(c) a calculation of the actual values for $r$ and $e$ was carried out. The residual deviations from the best-fitting asphere are shown in Fig. 7(d). 
a
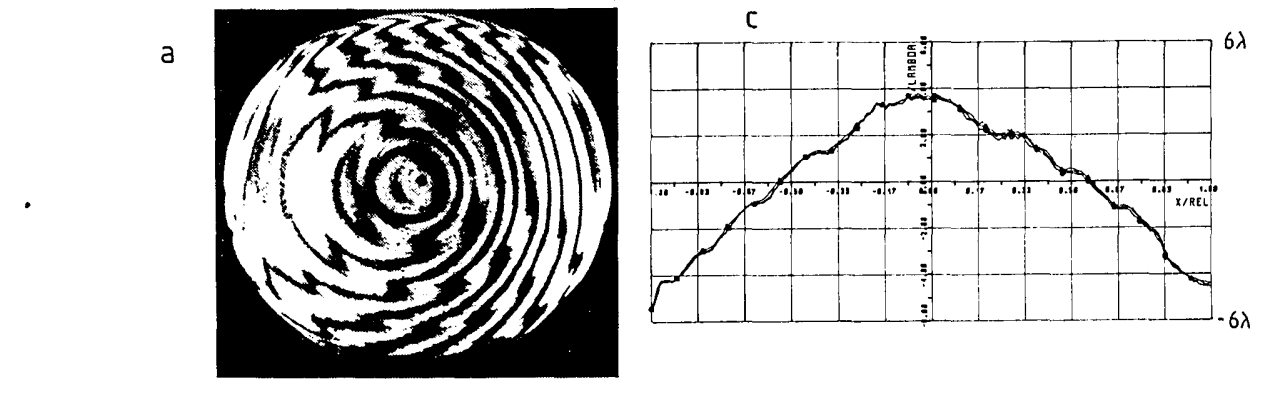

$U_{1}=1$

$U_{5}=2 r^{2}-1$

$U_{13}=6 r^{4}-6 r^{2}+1$

$U_{25}=20 r^{6}-30 r^{4}+12 r^{2}-1$

$U_{41}=70 r^{8}-140 r^{6}+90 r^{4}-20 r^{2}+1$

$U_{61}=252 r^{10}-630 r^{8}+560 r^{6}-210 r^{4}+30 r^{2}-1$
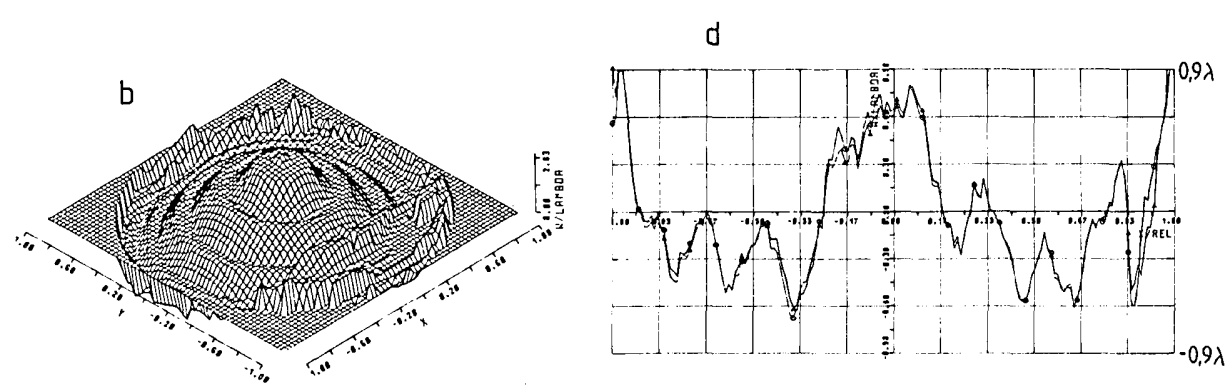

Fig. 7. (a) Interferogram of aspheric Ge surface; (b) 2-D analysis of wave front; (c) cross section through wave front from (b); (d) deviations from best-fitting asphere.

\section{Conclusion}

A Twyman-Green arrangement to test aspheric surfaces using partial lens compensation and computergenerated holograms has been described. Auxiliary lenses used to adapt the aperture of the test surface as well as to compensate some of the asphericity and to image the test surface onto the CGH need not be perfect when the interferometer is calibrated with the help of a well-known precise spherical mirror. In this case automatic fringe analysis is used to measure the error wave front arising from imperfect components and adjustment errors within the setup. A corrected hologram is then generated to compensate these errors. Fringe analysis has been carried out by a static method including tilt of the reference mirror to introduce a carrier frequency from which the wave front can be calculated by Fourier transform algorithms. A threephase step method has been reported allowing closed fringes to be evaluated. Taking into consideration that only odd terms arise when the test surface or the CGH is tilted or decentered, Zernike polynomials can be used to communicate with a ray tracing program to calculate the actual adjustment state. Since mostly small errors are introduced, linearity will hold leading to fast results of tilt and decentering errors, whose influence can be compensated by subtracting the odd terms.
Shape errors of the surface under test, such as errors of vertex radius, eccentricities, and higher-order asphericities, are detected in the same fashion using the rotational symmetric terms of the Zernike expansion.

This research was supported by a BMFT grant.

\section{References}

1. J. C. Wyant and V. P. Bennett, "Using Computer Generated Holograms to Test Aspheric Wavefronts," Appl. Opt. 11, 2833 (1972).

2. A. F. Fercher and M. Kriese, "Binäre synthetische Hologramme zur Prüfung asphärischer optischer Elemente," Optik 35, 168 (1972).

3. M. Foulde, A. F. Fercher, R. Torge, and R. N. Wilson, "Optical Testing by Means of Synthetic Holograms and Partial Lens Compensation," Opt. Commun. 7, 363 (1973).

4. J. C. Wyant and P. K. O'Neill, "Computer Generated Hologram; Null Lens Test of Aspheric Wavefronts," Appl. Opt. 13, 2762 (1974).

5. R. S. Sirohi, H. Blume, and K.-J. Rosenbruch, "Optical Testing Using Synthetic Holograms," Opt. Acta 23, 229 (1976).

6. A. F. Fercher, "Computer-Generated Holograms for Testing Optical Elements: Error Analysis and Error Compensation," Opt. Acta 23, 347 (1976).

7. A. Handojo and J. de Jong, "Interferometer for Optical Testing with Computer-Generated Holograms," Appl. Opt. 16, 546 (1977). 
8. T. Yatagai and H. Saito, "Interferometric Testing with Computer-Generated Holograms: Aberration Balancing Method and Error Analysis," Appl. Opt. 17, 558 (1978).

9. J. Schwider, R. Burow, and J. Grzanna, "CGH-Testing of Rotational-Symmetric Aspheric in Compensated Interferometers," Opt. Appl. 9, 39 (1979).

10. J. Schwider and R. Burow, "Wave Aberrations Caused by Misalignments of Aspherics and Their Elimination," Opt. Appl. 9, 33 (1979).

11. H. J. Tiziani, "Prospects of Testing Aspheric Surfaces with Computer-Generated Holograms," Proc. Soc. Photo-Opt. Instrum. Eng. 235, 72 (1980).

12. J. Schwider, J. Grzanna, R. Spolaczyk, and R. Burow, "Testing Aspherics in Reflected Light Using Blazed Synthetic Holograms," Opt. Acta 27, 683 (1980).

13. N. P. Larionov, A. V. Lukin, and R. A. Rafikov, "Testing of Aspherical Surfaces by Means of Axial Synthesized Holograms," Sov. J. Opt. Technol. 47, 667 (1980).

14. R. Mercier and S. Lowenthal, "Comparison of In-Line and Carrier Frequency Holograms in Aspheric Testing," Opt. Commun. 33, 251 (1980).

15. J. C. Wyant, "Testing Aspherics Using Two-Wavelength Holography," Appl. Opt. 10, 2113 (1971).

16. J. D. Rancourt, "Interferometric Fringe Analysis," in Optical Shop Notebook IX, J. B. Houston, Jr, Ed. (Optical Society of America, Washington, D.C., 1975) p. 69.

17. E. R. Freniere, O. E. Toler, and R. Race, "Interferogram Evaluation Program for the HP-9825A Calculator," Opt. Eng. 20, 253 (1981).

18. W. Augustyn, "Versatility of Microprocessor-Based Interferometric Data Reduction System," Proc. Soc. Photo-Opt. Instrum. Eng. 192, 128 (1979).

19. P. F. Forman, "The Zygo Interferometer System," Proc. Soc. Photo-Opt. Instrum. Eng. 192, 41 (1979).

20. W. R. J. Funnell, "Image Processing Applied to the Interactive Analysis of Interferometric Fringes," Appl. Opt. 20, 3245 (1981).

21. T. Yatagai, S. Nakadate, M. Idesawa, and H. Saito, "Automatic Fringe Analysis Using Digital Image Processing Techniques," Opt. Eng. 21, 432 (1982).

22. G. E. Bernal and J. Loomis, "Interactive Video Image DigitizerApplication to Interferogram Analysis," Proc. Soc. Photo-Opt. Instrum. Eng. 126, 143 (1977).

23. M. Takeda, H. Ina, and S. Kobayashi, "Fourier-Transform Method of Fringe-Pattern Analysis for Computer-Based Topography and Interferometry," J. Opt. Soc. Am. 72, 156 (1982).

24. Y. Ichioka and M. Inuiya, "Direct Phase Detecting System," Appl. Opt. 11, 1507 (1972).

25. L. Mertz, "Real-Time Fringe-Pattern Analysis," Appl. Opt. 22, 1535 (1983).

26. J. H. Bruning, D. R. Herriott, J. E. Gallagher, D. P. Rosenfeld, A. D. White, and D. J. Brangaccio, "Digital Wavefront Measuring Interferometer for Testing Optical Surfaces and Lenses," Appl. Opt. 13, 2693 (1974).

27. B. Dörband, "Die 3-Interferogramm-Methode zur automatischen Streifenauswertung in rechnergesteuerten digitalen Zweistrahlinterferometern," Optik 60, 156 (1982).

28. L. M. Frantz, A. A. Sawchuk, and W. von der Ohe, "Optical Phase Measurement in Real Time," Appl. Opt. 18, 3301 (1979).

29. J. Schwider, R. Burow, K.-E. Elssner, J. Grzanna, R. Spolaczyk, and K. Merkel, "Digital Wave-Front Measuring Interferometry: Some Systematic Error Sources,” Appl. Opt. 22, 3421 (1983).

30. N. A. Massie, "Real-Time Digital Heterodyne Interferometry: a System," Appl. Opt. 19, 154 (1980).

31. D. T. Moore, R. P. Murray, and F. B. Neves, "Large Aperture ac Interferometer for Optical Testing," Appl. Opt. 173959 (1978).
Books continued from page 2599

are also calculated. Finally, Chap. 11 examines field problems in gravitational fields. Examples are given of optical rotation sensors that are important in inertial navigation systems in aircraft and spacecraft and also in surveying. The mechanical gyroscope is now being replaced by an optical device where laser light, forced to travel in a circle, substitutes for the mechanical rotor. The result is an instrument based on relativistic ideas, which, without moving parts, gives a resolution of the order of $10^{-150} / \mathrm{h}$.

On the whole this book is of very high quality, and the formulas and equations give the impression of high mathematical exactness. Central to the work is the electrodynamics of moving bodies, which is of great relevance in telecommunications, e.g., in the interpretation of Doppler spectra from moving radar targets and to electric machine design. Relativity allows formulation of these problems, in a clear and precise fashion.

It is stated in the Preface that "the applicational approach used in the text should be acceptable to space engineers, nuclear engineers, electrical engineers and more generally applied physicists." I agree with this statement but as an engineer I must stress that this is a book of mathematical equations that are not that easy to understand or use in practice. I also find it rather frustrating that no solutions are given to the 118 problems presented.

It is also stated that the text has been written at the first-year graduate-student level and assumes an intermediate mathematical background as well as a reasonable foundation in electromagnetic theory. Again I want to point out that a great deal of mathematical and theoretical knowledge is needed to absorb the useful information that is presented in this interesting book. The list of some hundred references is impressive and will certainly be useful for deeper penetration into the subject.

\section{NILS ABRAMSON}

Self Organization and Management of Social Systems: Insights, Promises, Doubts, and Questions. Edited by H. ULRICH and G. B. PROBST. Springer-Verlag, New York, 1984. 155 pp. $\$ 23.00$.

Fluctuations and Sensitivity in Nonequilibrium Systems. Edited by W. HORSTEMKE and D. K. KONDEPUDI. SpringerVerlag, New York, 1984. 273 pp. $\$ 28.00$.

This is a review of two recent publications from Springer.

SELF-ORGANIZATION AND MANAGEMENT OF SOCIAL SYSTEMS is a postsymposium report of a conference held at the University of St. Gall, Switzerland, 14-16 Sept. 1983. It is yet another in the series on Synergetics. The volume might have been entitled "whither cybernetics"; it contains nothing of specific interest to the workers in the physical sciences. Nonetheless, I enjoyed reading the book, especially the lead article by Von Foerster. There were clear common threads throughout and considerable candor.

FLUCTUATIONS AND SENSITIVITY IN NONEQUILIBRIUM SYSTEMS is billed as a Proceeding in Physics 1, suggesting the initiation of yet another series from Springer. This book comes from a conference held in Austin, Tex., 12-16 Mar. 1984. There are optics articles from Rosenberger et al. and Abraham, both covering material that can be found elsewhere. For those in optics who have a general interest in nonlinear systems dynamics or special interests in nonequilibrium bistability and chaos, this book may be of interest. I have found it to be quite useful. It covers a wide range of problems that have parallels in optics. It is highly technical, and there are neither decent overviews nor outstanding individual articles, but the general level of presentation is comprehensible. There are several articles on the effects of colored noise, which is of current interest to many people working in laser physics.

\section{FREDERIC A. HOPF} continued on page 2620 